\section{PARENTAL PERCEPTION OF STAFF-PARENT COMMUNICATION IN THE NEONATAL UNIT}

\author{
K. Willis, P. Nath, C. Thurlow, M. James
}

Department of Paediatrics, Ipswich Hospital NHS Trust, Ipswich, UK

Background and aim: A high standard of communication between staff and parents in the neonatal unit is a vital part of family centered care. It is essential to the establishment of good working relationships and improves parent-baby bonding. Information provided should be free of medical jargon and presented in a way that parents can easily understand. We aimed to study parents' perception of the quality of communication in the neonatal unit.

Method: A twelve point questionnaire was sent by post to the parents of babies admitted to the neonatal unit from August - October 09.

Results: A total of 134 parents were approached and $74(55 \%)$ responded. Of the responses 50 $(68 \%)$ were from mothers and $22(30 \%)$ were from both parents. Gestational age ranged from $28-43$ weeks (Median 39.5 weeks) and birth weight from 592 - 5020 grams (Median 3165 grams).

$59(80 \%)$ had communication with medical staff within 24 hours of admission. 67 (91\%) parents were regularly updated during their baby's stay. When asked about the person updating, 40 (60\%) were regularly updated by the nurses and 15 $(22 \%)$ by both nursing and medical staff. $68(92 \%)$ parents were happy with the time spent, ease of understanding and sensitivity of conveyance of the information. $66(89 \%)$ reported overall satisfaction with the communication, $6(8 \%)$ were unsatisfied.

Conclusions: The majority of parents were regularly updated and overall were happy with the quality of communication in the neonatal unit. Parents identified nurses as the key source of information.

\section{8}

CONTEXTUAL FACTORS INFLUENCING PAIN RESPONSE TO HEEL STICK PROCEDURE IN PRETERM INFANTS: WHAT DO WE KNOW? A SYSTEMATIC REVIEW

\author{
G. Sellam 1 , E. Cignacco², S. Engberg ${ }^{3}$
}

${ }^{1}$ Institute of Nursing Sciense, ${ }^{2}$ Institute of Nursing Science, University of Basel, Basel, Switzerland,

${ }^{3}$ School of Nursing, University of Pittsburgh, Pittsburgh, PA, USA

Background: Major efforts to develop objective measurement tools for neonatal pain assessment have been made. However, the challenge of measuring pain in neonates remains, suggesting that contextual factors (CFs) might alter pain response in neonates. Although the role of CFs is increasingly discussed as crucial for pain assessment, they are not well described in the literature and barely considered in the clinical setting.

Aim: To explore the impact of CFs on pain response in preterm infants.

Method: Literature search was undertaken considering the period from 1990-2009. Studies reporting about the relation between one or more CFs and pain response in preterm infants during heel stick procedure were considered.

Results: 23 studies were included. The studies varied highly related to the design, sample, analysis procedures, and examined variables. Six categories of CFs emerged: Age, pain exposure, health status, therapeutic interventions, behavioral status, and demographic factors, indicating that some of the CFs might have an effect on pain response in preterms. However, none of the CFs was consistently related to pain response as evidenced by contradictory findings. Inconsistency between behavioral and physiological pain response related to CFs further aggravates accurate conclusions.

Conclusion: This review indicates a possible influence of some CFs on pain response. However, the results remain inconclusive, which is particularly due to the heterogeneity of the studies. CFs need further investigations in order to get more evidence regarding the magnitude of their effect on pain response. Further evidence has the potential to contribute to achieve another level of measurement of this complex phenomenon. 